\title{
Synthesis and Antimicrobial Activity of New Schiff Base Compounds Containing 2-Hydroxy-4-pentadecylbenzaldehyde Moiety
}

\author{
Gadada Naganagowda, ${ }^{1}$ Reinout Meijboom, ${ }^{1}$ and Amorn Petsom ${ }^{2}$ \\ ${ }^{1}$ Research Centre for Synthesis and Catalysis, Department of Chemistry, University of Johannesburg, \\ P.O. Box 524, Auckland Park, Johannesburg 2006, South Africa \\ ${ }^{2}$ Department of Chemistry, Faculty of Science, Chulalongkorn University, Bangkok 10330, Thailand
}

Correspondence should be addressed to Gadada Naganagowda; ngchula.pdf@gmail.com

Received 8 April 2014; Accepted 5 June 2014; Published 21 July 2014

Academic Editor: Atsushi Ohtaka

Copyright (C) 2014 Gadada Naganagowda et al. This is an open access article distributed under the Creative Commons Attribution License, which permits unrestricted use, distribution, and reproduction in any medium, provided the original work is properly cited.

Various novel Schiff base compounds have been synthesized by reaction of 2-hydroxy-4-pentadecylbenzaldehyde with substituted benzothiophene-2-carboxylic acid hydrazide and different substituted aromatic or heterocyclic amines in the presence of acetic acid in ethanol. The structures of all these compounds were confirmed by elemental analysis, IR, ${ }^{1} \mathrm{H}-\mathrm{NMR},{ }^{13} \mathrm{C}-\mathrm{NMR}$, and mass spectral data and have been screened for antibacterial and antifungal activity.

\section{Introduction}

Compounds with the structure of $-\mathrm{C}=\mathrm{N}-$ (azomethine group) are known as Schiff bases, which are usually synthesized by condensation of primary amines and active carbonyl groups. Schiff bases are an important class of compounds in the medicinal and the pharmaceutical field, including antibacterial $[1,2]$, antifungal $[3,4]$, and antitumor activity $[5,6]$. Heterocyclic-containing Schiff bases can show dramatically increased biological activities. As evident from literature [7], it was noted that a lot of research has been carried out on Schiff bases, but no work has been done on this particular type of Schiff base. Aromatic Schiff bases possessing long alkyl chains have received overwhelming attention due to their possibility to show mesomorphic properties such as smectic and nematic phases [8-11]. In this paper, we report the synthesis of a novel Schiff base from substituted benzothiophene-2-carboxylic acid hydrazide, various aromatic and heterocyclic amines with 2-hydroxy-4pentadecylbenzaldehyde as a moiety. We also report the results of biological screening for possible antibacterial and antifungal activity of the resulting derivatives and we discuss the relationship of molecular structure and the bioactivity (Table 1).

\section{Results and Discussion}

Cashew nut shell liquid (CNSL) is obtained as a by-product from mechanical processing for edible use of cashew kernel (Anacardium occidentale L.) and is a mixture of anacardic acid, cardanol, and smaller amounts of cardol and 2methyl cardol. Due to the easy thermal decarboxylation of anacardic acid, the main component of distilled CNSL is cardanol (yield up to $70-80 \%$ and purity up to $90 \%$ ) as a mixture of saturated (3-n-pentadecylphenol), monoolefinic [3-(n-pentadeca-8-enyl)phenol], diolefinic [3-(n-pentadeca8,11-dienyl)phenol], and triolefinic [3-(n-pentadeca-8,11,14trienyl)phenol] long-chain phenols, with an average value of two double bonds per molecule. Cardol and methyl cardol are present in smaller percentages (Figure 1).

Cardanol is a phenolic compound with a $\mathrm{C}_{15}$ aliphatic chain in the meta position, obtained from cashew nut shell liquid (CNSL). The structure and composition of cardanol is 


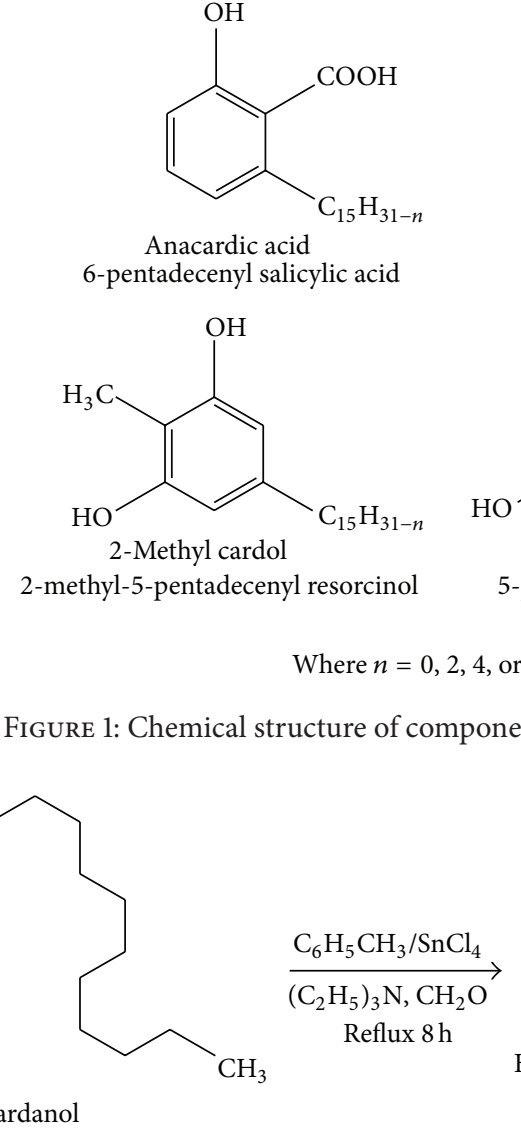

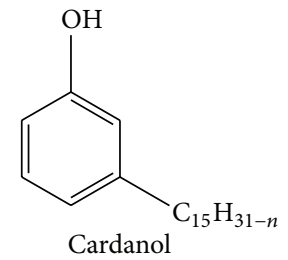

3-pentadecenyl phenol

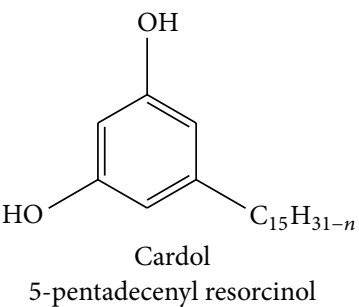

5-pentadecenyl resorcinol
1<smiles>CCCCCCCCCCCCCCCc1cccc(O)c1</smiles>

SCHEME 1: Synthesis of 2-hydroxy-6-pentadecylbenzaldehyde.
TABLE 1: Antibacterial and antifungal activity of the tested compounds.

\begin{tabular}{lcccc}
\hline \multirow{2}{*}{ Compound } & \multicolumn{4}{c}{ Zone of inhibition (mm) } \\
& S. aureus & E. coli & C. albicans & C. pannical \\
\hline 3a & 13.21 & 09.14 & 09.21 & 14.03 \\
3b & 08.24 & 08.91 & 08.21 & 12.11 \\
3c & 10.22 & 16.21 & 06.21 & 10.11 \\
3d & 15.12 & 16.11 & 07.34 & 12.23 \\
5a & 14.10 & 16.08 & 12.10 & 10.22 \\
6a & 13.25 & 14.20 & 16.12 & 13.20 \\
7a & 12.22 & 16.10 & 13.12 & 18.15 \\
8a & 16.14 & 13.10 & 09.10 & 12.13 \\
9b & 19.12 & 18.20 & 09.88 & 14.10 \\
9g & 17.20 & 16.10 & 10.21 & 12.10 \\
10a & 15.10 & 14.23 & 13.10 & 15.20 \\
11b & 14.12 & 13.12 & 15.10 & 14.13 \\
DMF (control) & 00 & 00 & 00 & 00 \\
Streptomycin & 24.30 & 22.12 & - & - \\
Griseofulvin & - & - & 21.05 & 20.13 \\
\hline
\end{tabular}

given in Figure 2. It is a mixture of saturated and unsaturated (mono-, di-, and tri-) compounds.
2-Hydroxy-4-pentadecylbenzaldehyde was prepared from cardanol (3-pentadecylphenol contained, e.g., in cashew nut shell liquid (CNSL)) by formylation, using a standard procedure [12] (Scheme 1).

Substituted benzothiophene was prepared by the reaction of aromatic cinnamic acid with thionyl chloride in DMF and dry pyridine according to the reported method [13]. Substituted benzothiophene-2-carbonyl chloride was then treated with hydrazine hydrate to obtain benzothiophene2-carbohydrazides 3a-d. Compounds $\mathbf{4 a}, \mathbf{4 b}, \mathbf{4 c}$, and $\mathbf{4 d}$ were synthesized by reaction of 2-hydroxy-6-pentadecylbenzaldehyde with 6-substitued-3-chloro-1-benzothiophene2 -carboxylicacidhydrazide in the presence of acetic acid in ethanol under reflux (Scheme 2).

Compounds $\mathbf{5} \mathbf{a}$ and $\mathbf{6} \mathbf{a}$ were obtained separately in good yield by refluxing compound 2 with an equimolar amount of compounds $\mathbf{5}$ and $\mathbf{6}$ in the presence of acetic acid in ethanol for $5 \mathrm{~h}$. The configuration at the imine unit was not investigated; compounds $\mathbf{5} \mathbf{a}$ and $\mathbf{6} \mathbf{a}$ are arbitrarily shown in Z configuration in Scheme 3.

2-Hydroxy-4-pentadecylbenzaldehyde 2 was treated with aromatic amines to get new cardanol aldehyde derivative of Schiff base compounds. These new Schiff bases were fully characterized by elemental analysis, IR, MS, ${ }^{1} \mathrm{H}$, and ${ }^{13}$ C-NMR data. 
<smiles>C=CC/C=C\C/C=C/CCCCCCCc1cccc(O)c1</smiles><smiles>CCCCCC/C=C/CCCCCCCc1cccc(O)c1</smiles>

FIgURE 2: Structure and composition of cardanol.<smiles>CCCCCCCCCCCCCCCc1ccc(C=O)c(O)c1</smiles>

2

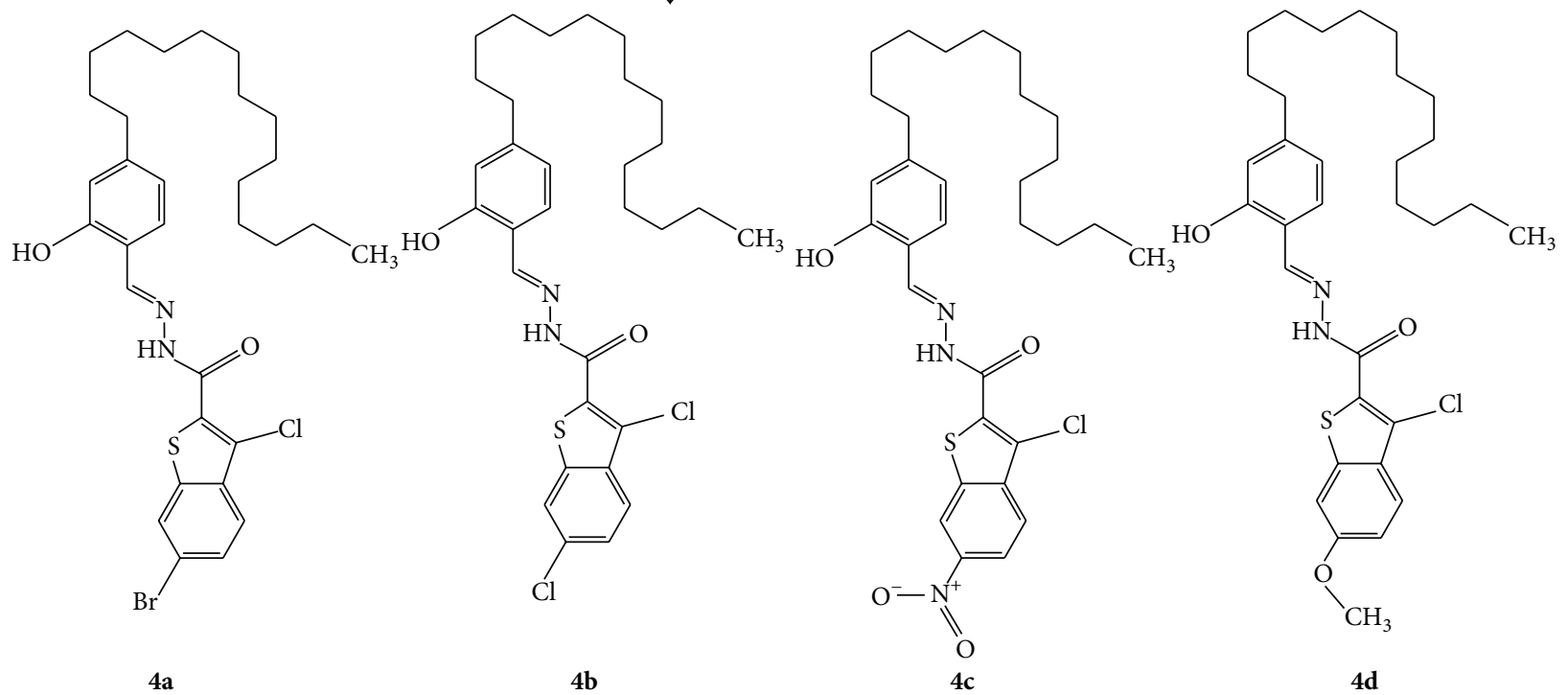

Scheme 2: Synthesis of 6-substitued-3-chloro-N'-(2-hydroxy-4-pentadecylbenzylidene)benzothiophene-2-carbohydrazide. 


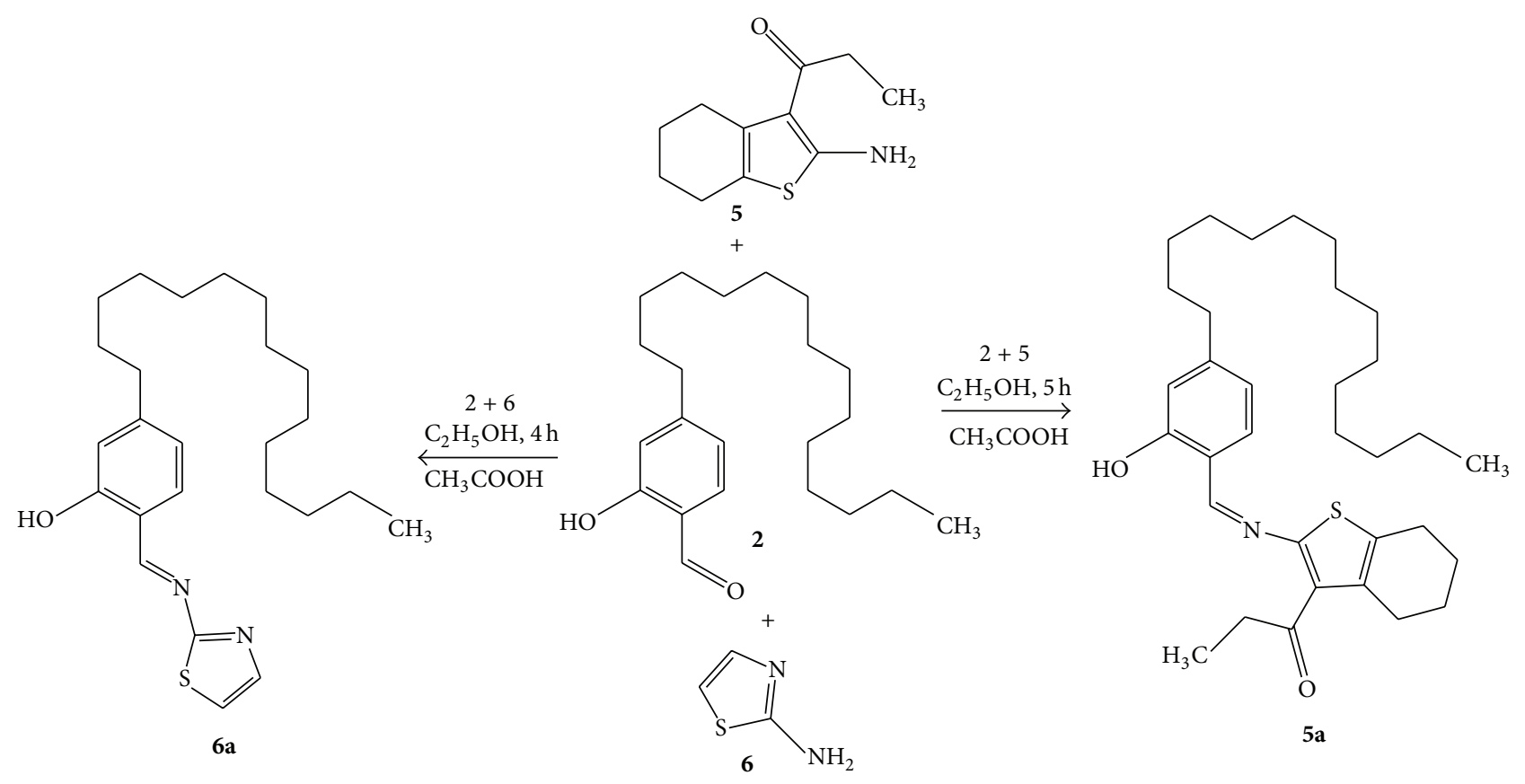

Scheme 3: Synthesis of new heterocyclic Schiff base compounds.

\section{Antimicrobial Evaluation}

The cup plate method using Hi-Media agar medium was employed to study the antibacterial activity of the synthesized compounds against Gram-positive bacteria, Staphylococcus aureus, and Gram-negative bacteria, Escherichia coli. Preparation of nutrient broth, subculture, base layer medium, agar medium, and peptone water was carried out according to a standard procedure [14]. Streptomycin was used as a reference drug and dimethylformamide as a control. The zone of inhibition produced by each compound was measured in $\mathrm{mm}$. The evaluated compounds have shown low to moderate activities as compared to standard drug against the bacteria. The antifungal activities of the synthesized compounds were evaluated against two different fungi, that is, Candida albicans and Chrysosporium pannical, by the filter paper disc technique [15].

\section{Experimental}

All chemicals were analytical grade, purchased from commercial suppliers, and used as received without further purification. Melting points were determined in open capillaries and are uncorrected. FT-IR spectra were recorded on a Nicolet Fourier Transform IR spectrophotometer Impact 410 (Nicolet Instrument Technologies, Inc. WI, USA). ${ }^{1} \mathrm{H}-$ NMR and ${ }^{13} \mathrm{C}-\mathrm{NMR}$ spectra were obtained in DMSO- $d_{6}$ at $400 \mathrm{MHz}$ for ${ }^{1} \mathrm{H}$ nuclei and $100 \mathrm{MHz}$ for ${ }^{13} \mathrm{C}$ nuclei (Bruker Company, Germany). All chemical shifts are expressed in ppm relative to tetramethylsilane (TMS) as the internal standard. Mass spectra were obtained using matrix-assisted laser desorption ionization mass spectrometry (MALDITOF) by using dithranol as a matrix. Elemental analysis
(C, H, N, and S) was performed on a Perkin Elmer 2400 analyzer. The purity of compounds was checked by TLC on silica gel and further purification was performed using column chromatography (silica gel, 60-120 mesh).

4.1. Synthesis of Compound 2 Was Prepared according to Literature Procedure. See [12].

4.2. Synthesis of Compounds $\mathbf{3} \boldsymbol{a}$ - $\boldsymbol{d}$ Was Also Prepared according to Literature Procedure. See [13].

4.3. General Procedure for Synthesis of Compounds $\mathbf{4 a - d :}$ Detail for Preparation of 6-Bromo-3-chloro- $N^{\prime}-(2-h y d r o x y-4-$ pentadecylbenzylidene)benzo[b]thiophene-2-carbohydr azide (4a). 2-Hydroxy-6-pentadecylbenzaldehyde 2 (3.32 g, $0.01 \mathrm{~mol}$ ) was treated with 6-bromo-3-chloro-1-benzothiophene-2-carboxylicacidhydrazide $3 \mathrm{a}(3.03 \mathrm{~g}, 0.01 \mathrm{~mol})$ in the presence of glacial acetic acid $(1 \mathrm{~mL})$ in absolute ethanol $(25 \mathrm{~mL})$ and was heated under reflux for $5 \mathrm{~h}$. The completion of the reaction was monitored by TLC. The reaction mixture was allowed to cool down to room temperature and then poured onto crushed ice. The precipitate was filtered, dried, and recrystallized from absolute ethanol. The resulting solid was further purified by column chromatography [silica, petroleum ether/ethyl acetate $(85: 15)]$, leading to compound $\mathbf{4 a}$ as yellow solid.

Yield 71\%; mp 245-247 C; IR $v\left(\mathrm{~cm}^{-1}\right)$ : 3431 (OH st), 3181 (N-H st), 1645 (C=O st), 1586 (C=N st); ${ }^{1} \mathrm{H}-\mathrm{NMR} \delta$ (ppm): $12.10(\mathrm{~s}, 1 \mathrm{H}, \mathrm{OH}), 11.34(\mathrm{~s}, 1 \mathrm{H}, \mathrm{NH}), 8.66(\mathrm{~s}, 1 \mathrm{H}, \mathrm{CH}=\mathrm{N})$, 8.12 (s, 1H, Ar-H), 8.01 (d, J = $7.8 \mathrm{~Hz}, 1 \mathrm{H}, \mathrm{Ar}-\mathrm{H}), 7.55$ $(\mathrm{d}, J=7.8 \mathrm{~Hz}, 1 \mathrm{H}, \mathrm{Ar}-\mathrm{H}), 7.46(\mathrm{~d}, J=7.6 \mathrm{~Hz}, 1 \mathrm{H}, \mathrm{Ar}-$ $\mathrm{H}), 6.95(\mathrm{~s}, 1 \mathrm{H}, \mathrm{Ar}-\mathrm{H}), 6.60(\mathrm{~d}, J=6.8 \mathrm{~Hz}, 1 \mathrm{H}, \mathrm{Ar}-\mathrm{H})$, 
$2.52\left(\mathrm{t}, J=7.6 \mathrm{~Hz}, 2 \mathrm{H}, \mathrm{Ar}-\mathrm{CH}_{2}\right), 1.54-1.01\left(\mathrm{~m}, 26 \mathrm{H},\left(\mathrm{CH}_{2}\right)_{13}\right)$, $0.85\left(\mathrm{t}, J=6.8 \mathrm{~Hz}, 3 \mathrm{H}, \mathrm{CH}_{3}\right) ;{ }^{13} \mathrm{C}-\mathrm{NMR} \delta(\mathrm{ppm}): 169.1$ $(\mathrm{CONH}), 160.9,154.0$ (aromatic carbons), $146.0(\mathrm{CH}=\mathrm{N})$, 145.4, 141.6, 132.4, 130.4, 129.8, 129.3, 125.4, 125.4, 120.3, 119.0, 117.2 , 115.5 (aromatic carbons), 35.3, 31.2, 30.2, 30.1, 28.9, 28.9, 28.8, 28.7, 28.6, 28.5, 22.0 (methylene carbons), $13.8\left(\mathrm{CH}_{3}\right)$; MS, $m / z: 621.65\left(\mathrm{M}^{+}\right)$. Anal. calcd. for $\mathrm{C}_{31} \mathrm{H}_{40} \mathrm{ClBrN}_{2} \mathrm{O}_{2} \mathrm{~S}: \mathrm{C}$, 60.05; H, 6.50; N, 4.52; S, 5.17; found: C, 60.04; H, 6.82; N, 4.39 ; S, $4.82 \%$.

Similarly, the compounds $\mathbf{4 b}$-d were prepared with little change in reflux time and reaction work up.

4.3.1. 3,6-Dichloro- $N^{\prime}$-(2-hydroxy-4-pentadecylbenzylidene) benzo[b] thiophene-2-carbohydrazide (4b). Yield 68\%; mp 280-282 C; IR $v\left(\mathrm{~cm}^{-1}\right): 3426$ (OH st), 3168 (N-H st), 1640 (C=O st), 1581 (C=N st); ${ }^{1} \mathrm{H}-\mathrm{NMR} \delta$ (ppm): 11.80 (s, $\left.1 \mathrm{H}, \mathrm{OH}\right)$, 11.68 (s, 1H, NH), $8.51(\mathrm{~s}, 1 \mathrm{H}, \mathrm{CH}=\mathrm{N}), 8.09$ (s, 1H, Ar-H), 7.96 $(\mathrm{d}, J=7.8 \mathrm{~Hz}, 1 \mathrm{H}, \mathrm{Ar}-\mathrm{H}), 7.54(\mathrm{~d}, J=7.7 \mathrm{~Hz}, 1 \mathrm{H}, \mathrm{Ar}-\mathrm{H})$, $7.41(\mathrm{~d}, J=7.7 \mathrm{~Hz}, 1 \mathrm{H}, \mathrm{Ar}-\mathrm{H}), 6.95(\mathrm{~s}, 1 \mathrm{H}, \mathrm{Ar}-\mathrm{H}), 6.88(\mathrm{~d}$, $J=6.8 \mathrm{~Hz}, 1 \mathrm{H}, \mathrm{Ar}-\mathrm{H}), 2.55\left(\mathrm{t}, J=7.6 \mathrm{~Hz}, 2 \mathrm{H}, \mathrm{Ar}-\mathrm{CH}_{2}\right)$, $1.57-1.03\left(\mathrm{~m}, 26 \mathrm{H},\left(\mathrm{CH}_{2}\right)_{13}\right), 0.81\left(\mathrm{t}, J=6.8 \mathrm{~Hz}, 3 \mathrm{H}, \mathrm{CH}_{3}\right)$; ${ }^{13} \mathrm{C}-\mathrm{NMR} \delta$ (ppm): $169.4(\mathrm{CONH}), 160.9,153.2$ (aromatic carbons), $146.2(\mathrm{CH}=\mathrm{N}), 145.3,141.3,132.9,131.3,130.4,129.2$, $126.1,124.3,122.4,120.5,117.2,115.3$ (aromatic carbons), 36.3, 31.8, 31.2, 30.8, 29.7, 29.6, 29.6, 29.6, 29.5, 29.4, 29.3, 29.2, 22.7 (methylene carbons), $14.1\left(\mathrm{CH}_{3}\right) ; \mathrm{MS}, \mathrm{m} / z$ : $621.65\left(\mathrm{M}^{+}+1\right)$. Anal. calcd. for $\mathrm{C}_{31} \mathrm{H}_{40} \mathrm{Cl}_{2} \mathrm{~N}_{2} \mathrm{O}_{2} \mathrm{~S}$ : C, 64.68; H, 7.00; N, 4.87; S, 5.57; found: C, 64.04; H, 6.92; N, 4.39; S, 4.99\%.

4.3.2.3-Chloro- $N^{\prime}$-(2-hydroxy-4-pentadecylbenzylidene)-6-nitrobenzo[b] thiophene-2-carbohydrazide (4c). Yield 69\%; mp 291-293 ${ }^{\circ}$; IR $v\left(\mathrm{~cm}^{-1}\right): 3435$ (OH st), 3177 (N-H st), 1634 $\left(\mathrm{C}=\mathrm{O}\right.$ st), 1577 (C=N st); ${ }^{1} \mathrm{H}-\mathrm{NMR} \delta$ (ppm): 11.71 (s, $\left.1 \mathrm{H}, \mathrm{OH}\right)$, 11.62 (s, 1H, NH), 8.74 (s, 1H, CH=N), 8.02 (s, 1H, Ar-H), 7.98 $(\mathrm{d}, J=7.8 \mathrm{~Hz}, 1 \mathrm{H}, \mathrm{Ar}-\mathrm{H}), 7.61(\mathrm{~d}, J=7.8 \mathrm{~Hz}, 1 \mathrm{H}, \mathrm{Ar}-\mathrm{H})$, $7.47(\mathrm{~d}, J=7.6 \mathrm{~Hz}, 1 \mathrm{H}, \mathrm{Ar}-\mathrm{H}), 6.90$ (s, 1H, Ar-H), 6.77 (d, $J=6.8 \mathrm{~Hz}, 1 \mathrm{H}, \mathrm{Ar}-\mathrm{H}), 2.54\left(\mathrm{t}, J=7.6 \mathrm{~Hz}, 2 \mathrm{H}, \mathrm{Ar}-\mathrm{CH}_{2}\right)$, $1.55-1.01\left(\mathrm{~m}, 26 \mathrm{H},\left(\mathrm{CH}_{2}\right)_{13}\right), 0.88\left(\mathrm{t}, J=6.8 \mathrm{~Hz}, 3 \mathrm{H}, \mathrm{CH}_{3}\right)$; ${ }^{13} \mathrm{C}-\mathrm{NMR} \delta(\mathrm{ppm}): 167.2(\mathrm{CONH}), 160.4,152.7$ (aromatic carbons), $146.3(\mathrm{CH}=\mathrm{N}), 145.4,144.3,141.3,139.5,130.3,129.9$, $124.1,120.8,119.3,117.8,117.3,115.4$ (aromatic carbons), 36.4, 36.2, 31.9, 30.9, 30.6, 29.8, 29.7, 29.6, 29.5, 29.4, 29.3, 29.2, 22.7 (methylene carbons), 14.1 $\left(\mathrm{CH}_{3}\right)$; MS, $\mathrm{m} / z: 586.18\left(\mathrm{M}^{+}\right)$. Anal. calcd. for $\mathrm{C}_{31} \mathrm{H}_{40} \mathrm{ClN}_{3} \mathrm{O}_{4} \mathrm{~S}: \mathrm{C}, 63.52 ; \mathrm{H}, 6.88 ; \mathrm{N}, 7.17 ; \mathrm{S}, 5.47$; found: C, 62.44; H, 6.21; N, 6.99; S, 5.10\%.

4.3.3. 3-Chloro- $N^{\prime}$-(2-hydroxy-4-pentadecylbenzylidene)-6-methoxybenzo[b] thiophene-2-carbohydrazide (4d). Yield 81\%; $\mathrm{mp} 276-278^{\circ} \mathrm{C}$; IR $v\left(\mathrm{~cm}^{-1}\right): 3420$ (OH st), 3159 (N-H st), 1638 (C=O st), 1591 (C=N st); ${ }^{1} \mathrm{H}-\mathrm{NMR} \delta(\mathrm{ppm}): 12.03$ (s, $\left.1 \mathrm{H}, \mathrm{OH}\right)$, $11.81(\mathrm{~s}, 1 \mathrm{H}, \mathrm{NH}), 8.88(\mathrm{~s}, 1 \mathrm{H}, \mathrm{CH}=\mathrm{N}), 7.98(\mathrm{~d}, J=7.7 \mathrm{~Hz}$, $1 \mathrm{H}, \mathrm{Ar}-\mathrm{H}), 7.66$ (d, J = 7.6 Hz, 1H, Ar-H), 7.22 (s, 1H, Ar$\mathrm{H}), 7.02(\mathrm{~s}, 1 \mathrm{H}, \mathrm{Ar}-\mathrm{H}), 6.98(\mathrm{~d}, J=7.7 \mathrm{~Hz}, 1 \mathrm{H}, \mathrm{Ar}-\mathrm{H}), 6.90$ $(\mathrm{d}, J=6.8 \mathrm{~Hz}, 1 \mathrm{H}, \mathrm{Ar}-\mathrm{H}), 3.81\left(\mathrm{~s}, 3 \mathrm{H}, \mathrm{OCH}_{3}\right), 2.51(\mathrm{t}, J=$ $\left.7.6 \mathrm{~Hz}, 2 \mathrm{H}, \mathrm{Ar}-\mathrm{CH}_{2}\right), 1.56-1.01\left(\mathrm{~m}, 26 \mathrm{H},\left(\mathrm{CH}_{2}\right)_{13}\right), 0.88(\mathrm{t}, J=$ $\left.6.8 \mathrm{~Hz}, 3 \mathrm{H}, \mathrm{CH}_{3}\right) ;{ }^{13} \mathrm{C}-\mathrm{NMR} \delta$ (ppm): $168.3(\mathrm{CONH}), 160.5$, 159.4, 152.8 (aromatic carbons), $146.2(\mathrm{CH}=\mathrm{N}), 145.0,141.5$, $130.5,129.9,126.1,125.5,124.2,120.3,117.1,115.5,115.3$ (aromatic carbons), $55.5\left(\mathrm{OCH}_{3}\right), 36.1,31.8,31.2,29.9,29.8,29.7,29.6$, 29.5, 29.4, 29.3, 29.2, 22.7 (methylene carbons), $14.1\left(\mathrm{CH}_{3}\right)$; MS, $m / z: 570.21\left(\mathrm{M}^{+}+1\right)$. Anal. calcd. for $\mathrm{C}_{32} \mathrm{H}_{43} \mathrm{ClN}_{2} \mathrm{O}_{3} \mathrm{~S}: \mathrm{C}$, 67.29; H, 7.59; N, 4.87; S, 5.61; found: C, 67.04; H, 7.21; N, 4.20; S, $5.34 \%$.

4.4. Synthesis of Ethyl 2-(2-hydroxy-4-pentadecylbenzylideneamino)-4,5,6,7-tetrahydrobenzothiophene-3-carboxylate (5a). Ethyl-2-amino-4,5,6,7-tetrahydrobenzothiophene-3-carboxylate $5(2.08 \mathrm{~g}, 0.01 \mathrm{~mol})$ and 2-hydroxy-4-pentadecylbenzaldehyde $2(3.32 \mathrm{~g}, 0.01 \mathrm{~mol})$ in the presence of acetic acid $(1 \mathrm{~mL})$ in absolute ethanol $(25 \mathrm{~mL})$ were heated under reflux for $5 \mathrm{~h}$. It was then concentrated, cooled, and kept overnight in a refrigerator. The precipitate was filtered, washed with ethanol, dried, and recrystallized from methanol to get pure compound $\mathbf{5 a}$.

Yield: 66\%; mp: $234-236^{\circ} \mathrm{C}$; IR $(\mathrm{KBr}): \nu_{\max }\left(\mathrm{cm}^{-1}\right)$ : 3421.11 (O-H str.), 1611.61 (C=N str.), 1600 (C=O); ${ }^{1} \mathrm{H}-\mathrm{NMR}$ $\delta$ ppm: $12.10(\mathrm{~s}, 1 \mathrm{H}, \mathrm{OH}), 8.60(\mathrm{~s}, 1 \mathrm{H}, \mathrm{CH}=\mathrm{N}), 7.54(\mathrm{~d}, J=$ $7.8 \mathrm{~Hz}, 1 \mathrm{H}, \mathrm{Ar}-\mathrm{H}), 7.10$ (s, $1 \mathrm{H}, \mathrm{Ar}-\mathrm{H}), 6.89$ (d, $J=7.6 \mathrm{~Hz}$, $1 \mathrm{H}, \mathrm{Ar}-\mathrm{H}), 3.31\left(\mathrm{q}, 2 \mathrm{H}, \mathrm{COCH}_{2}\right), 2.71(\mathrm{t}, J=6.7 \mathrm{~Hz}$, $2 \mathrm{H}$, tetrahydrobenzothiophene), $2.62(\mathrm{t}, J=6.7 \mathrm{~Hz}, 2 \mathrm{H}$, tetrahydrobenzothiophene), $2.58(\mathrm{t}, J=7.6 \mathrm{~Hz}, 2 \mathrm{H}, \mathrm{Ar}-$ $\left.\mathrm{CH}_{2}\right), 1.81$ (m, $4 \mathrm{H}$, tetrahydrobenzothiophene), 1.59-1.31 (m, $\left.26 \mathrm{H},\left(\mathrm{CH}_{2}\right)_{13}\right), 1.25$ (q, 3H, $\mathrm{CH}_{3}$ Ester), 0.85 (t, $J=6.8 \mathrm{~Hz}, 3 \mathrm{H}$, $\left.\mathrm{CH}_{3}\right) ;{ }^{13} \mathrm{C}-\mathrm{NMR} \delta$ ppm: 189.6, 161.3, 156.8, 151.9, 143.4, 129.9, $128.6,120.3,118.9,118.3,116.0,115.0$ (aromatic carbons), 35.5, 35.4, 34.9, 31.5, 30.8, 30.1, 28.9, 28.8, 28.7, 28.6, 28.5, 23.2, 23.1, 22.9, 22.4, 22.3, 13.4 (methylene carbons), $14.2\left(\mathrm{CH}_{3}\right)$; $\mathrm{MS}$, $m / z: 538.45\left(\mathrm{M}^{+}\right)$. Anal. calcd. for $\mathrm{C}_{33} \mathrm{H}_{49} \mathrm{NO}_{3} \mathrm{~S}: \mathrm{C}, 73.42 ; \mathrm{H}$, 9.15; N, 2.59; S, 5.94 found: C, 73.14; H, 9.41; N, 2.13; S, 5.81\%.

4.5. Synthesis of 5-Pentadecyl-2-((thiazol-2-ylimino)methyl) phenol (6a). Cardanol aldehyde $2(3.32 \mathrm{~g}, 0.01 \mathrm{~mol})$ in ethanol $(15 \mathrm{~mL})$ and 2 -aminothiazole $(1.00 \mathrm{~g}, 0.01 \mathrm{~mol})$ in the presence of glacial acetic acid $(1 \mathrm{~mL})$ were refluxed for $6 \mathrm{~h}$. The reaction mixture was allowed to cool down to room temperature and poured onto ice cooled water with constant stirring. The resulting precipitate was filtered, washed with water, dried, and purified through column chromatography to get pure compound $\mathbf{6 a}$ as light yellowish solid.

Yield: $51 \%$; mp: $220-222^{\circ} \mathrm{C}$; IR $(\mathrm{KBr}): v_{\max }\left(\mathrm{cm}^{-1}\right): 3189.31$ (O-H str.), 1612.77 (C=N str.); ${ }^{1} \mathrm{H}-\mathrm{NMR} \delta$ ppm: 11.80 (s, $1 \mathrm{H}$, $\mathrm{OH}), 8.65(\mathrm{~s}, 1 \mathrm{H}, \mathrm{CH}=\mathrm{N}), 7.85(\mathrm{~d}, J=7.3 \mathrm{~Hz}, 1 \mathrm{H}$, thiazole ring), $7.65(\mathrm{~d}, J=7.8 \mathrm{~Hz}, 1 \mathrm{H}, \operatorname{Ar}-\mathrm{H}), 7.60(\mathrm{~d}, J=7.3 \mathrm{~Hz}$, $1 \mathrm{H}$, thiazole ring), $7.06(\mathrm{~s}, 1 \mathrm{H}, \mathrm{Ar}-\mathrm{H}), 6.85(\mathrm{~d}, J=7.6 \mathrm{~Hz}, 1 \mathrm{H}$, $\mathrm{Ar}-\mathrm{H}), 2.68\left(\mathrm{t}, J=7.6 \mathrm{~Hz}, 2 \mathrm{H}, \mathrm{Ar}-\mathrm{CH}_{2}\right), 1.61-1.33(\mathrm{~m}, 26 \mathrm{H}$, $\left.\left(\mathrm{CH}_{2}\right)_{13}\right), 0.88\left(\mathrm{t}, J=6.8 \mathrm{~Hz}, 3 \mathrm{H}, \mathrm{CH}_{3}\right) ;{ }^{13} \mathrm{C}-\mathrm{NMR} \delta \mathrm{ppm}$ : $162.3,157.0,152.2,143.9,128.9,128.6,121.7,119.0,118.4,116.1$, 114.8 (aromatic carbons), 35.3, 35.1, 34.8, 31.2, 30.4, 30.0, 28.9, 28.8, 28.7, 28.6, 28.5, 28.4, 22.9, 14.5 (methylene carbons), 14.2 $\left(\mathrm{CH}_{3}\right)$; MS, $m / z$ : $413.13\left(\mathrm{M}^{+}\right)$. Anal. calcd. for $\mathrm{C}_{25} \mathrm{H}_{38} \mathrm{~N}_{2} \mathrm{OS}$ : C, 72.42; H, 9.24; N, 6.76; S; 7.73 found: C, 72.04; H, 9.14; N, 6.37; S, 7.81\%.

4.6. Synthesis of 2-((4-(Dimethylamino)phenylimino)methyl)5-pentadecylphenol $(7 \boldsymbol{a})$. To a stirred solution of compound $2(3.32 \mathrm{~g}, 0.01 \mathrm{~mol})$ and $\mathrm{N}, \mathrm{N}$-dimethylbenzene-1,4-diamine 
$(1.36 \mathrm{~g}, 0.01 \mathrm{~mol})$ in ethanol $(20 \mathrm{~mL})$, glacial acetic acid $(1 \mathrm{~mL})$ was added. The mixture was refluxed for $4 \mathrm{~h}$. After the completion of the reaction (TLC-monitoring) the reaction mixture was cooled down to room temperature and then added to crushed ice. The precipitate was filtered, washed with water, dried, and purified through column chromatography by using n-hexane and ethyl acetate $(50: 50)$ as an eluent to afford pure compound $7 \mathbf{a}$.

Yield: 58\%; mp: $291-221^{\circ} \mathrm{C}$; IR (KBr): $\nu_{\max }\left(\mathrm{cm}^{-1}\right): 321.06$ (O-H str.), 1619.34 (C=N str.); ${ }^{1} \mathrm{H}-\mathrm{NMR} \delta$ ppm: 12.01 (s, $1 \mathrm{H}$, $\mathrm{OH}), 8.73(\mathrm{~s}, 1 \mathrm{H}, \mathrm{CH}=\mathrm{N}), 7.60(\mathrm{~d}, J=7.8 \mathrm{~Hz}, 1 \mathrm{H}, \mathrm{Ar}-\mathrm{H})$, $7.52(\mathrm{~d}, J=7.7 \mathrm{~Hz}, 2 \mathrm{H}, \mathrm{Ar}-\mathrm{H}), 7.10$ (s, 1H, Ar-H), 6.96 (d, $J=7.8 \mathrm{~Hz}, 1 \mathrm{H}, \mathrm{Ar}-\mathrm{H}), 6.59(\mathrm{~d}, J=7.7 \mathrm{~Hz}, 2 \mathrm{H}, \mathrm{Ar}-\mathrm{H}), 3.01$ $\left(\mathrm{s}, 6 \mathrm{H}, \mathrm{H}_{3} \mathrm{C}-\mathrm{N}-\mathrm{CH}_{3}\right), 2.63\left(\mathrm{t}, J=7.6 \mathrm{~Hz}, 2 \mathrm{H}, \mathrm{Ar}-\mathrm{CH}_{2}\right), 1.61-$ $1.10\left(\mathrm{~m}, 26 \mathrm{H},\left(\mathrm{CH}_{2}\right)_{13}\right), 0.83\left(\mathrm{t}, J=6.8 \mathrm{~Hz}, 3 \mathrm{H}, \mathrm{CH}_{3}\right) ;{ }^{13} \mathrm{C}-$ NMR $\delta$ ppm: 160.9, 157.2, 152.7, 143.6, 130.8, 128.9, 122.4, 118.5, $115.0,114.7,112.5$ (aromatic carbons), 40.1, 35.3, 35.1, 34.8, 31.2, 30.8, 30.1, 28.9, 28.8, 28.7, 28.6, 28.5, 28.4, 27.3, 13.8 (methylene carbons), $13.5\left(\mathrm{CH}_{3}\right)$; MS, $m / z: 449.13$. $\left(\mathrm{M}^{+}\right)$. Anal. calcd. for $\mathrm{C}_{30} \mathrm{H}_{46} \mathrm{~N}_{2} \mathrm{O}$ : C, 79.95; $\mathrm{H}, 10.29 ; \mathrm{N}, 6.22$; found: C, 79.34; $\mathrm{H}$, $10.16 ; \mathrm{N}, 6.03 \%$.

4.7. Synthesis of 2-((4-Nitronaphthalen-1-ylimino)methyl)-5pentadecylphenol (8a). A mixture of compound 2 (3.32 g, $0.01 \mathrm{~mol}$ ) and 4-nitronaphthalen-1-amine (1.88 g, $0.01 \mathrm{~mol}$ ) in ethanol $(20 \mathrm{~mL})$ and few drops of glacial acetic acid was refluxed for $5 \mathrm{~h}$. After the completion of the reaction (TLC-monitoring), the reaction mixture was cooled down to room temperature and then poured into ice cold water. The precipitate was filtered, dried, and recrystallized from methanol to give pure product $\mathbf{8 a}$ as light yellowish solid.

Yield: 66\%; mp: $234-236^{\circ} \mathrm{C}$; IR (KBr): $\nu_{\max }\left(\mathrm{cm}^{-1}\right)$ : 3435.23 (O-H str.), 1624.07 (C=N str.); ${ }^{1} \mathrm{H}-\mathrm{NMR} \delta$ ppm: 12.03 $(\mathrm{s}, 1 \mathrm{H}, \mathrm{OH}), 8.90(\mathrm{~d}, J=7.8 \mathrm{~Hz}, 1 \mathrm{H}, \mathrm{Ar}-\mathrm{H}), 8.89(\mathrm{~s}, 1 \mathrm{H}$, $\mathrm{CH}=\mathrm{N}), 8.50(\mathrm{~d}, J=7.8 \mathrm{~Hz}, 1 \mathrm{H}, \mathrm{Ar}-\mathrm{H}), 8.20(\mathrm{~d}, J=7.8 \mathrm{~Hz}$, $1 \mathrm{H}, \operatorname{Ar}-\mathrm{H}), 7.85-7.75(\mathrm{~m}, 2 \mathrm{H}, \mathrm{Ar}-\mathrm{H}), 7.60(\mathrm{~d}, J=7.7 \mathrm{~Hz}$, 1H, Ar-H), 7.55 (s, 1H, Ar-H), 6.98 (s, 1H, Ar-H), 6.89 (d, $J=7.7 \mathrm{~Hz}, 1 \mathrm{H}, \mathrm{Ar}-\mathrm{H}), 2.63\left(\mathrm{t}, J=7.6 \mathrm{~Hz}, 2 \mathrm{H}, \mathrm{Ar}-\mathrm{CH}_{2}\right)$, $1.56-1.13\left(\mathrm{~m}, 26 \mathrm{H},\left(\mathrm{CH}_{2}\right)_{13}\right), 0.89\left(\mathrm{t}, J=6.8 \mathrm{~Hz}, 3 \mathrm{H}, \mathrm{CH}_{3}\right)$; ${ }^{13} \mathrm{C}-\mathrm{NMR} \delta$ ppm: $162.4,157.2,152.1,143.6,129.8,128.9,125.4$ $120.1,119.9,118.9,118.4,118.2,116.5,115.8,114.5,113.4$ (aromatic carbons), 35.3, 35.1, 34.8, 31.2, 30.8, 30.1, 28.9, 28.8, 28.7, 28.6, 28.5, 24.3, 23.2, 14.2 (methylene carbons), $13.5\left(\mathrm{CH}_{3}\right)$; $\mathrm{MS}$, $m / z: 502.23\left(\mathrm{M}^{+}+1\right)$. Anal. calcd. for $\mathrm{C}_{32} \mathrm{H}_{42} \mathrm{~N}_{2} \mathrm{O}_{3}$ : C, 76.46; $\mathrm{H}, 8.42$; N, 5.57; found: C, 76.04; H, 8.26; N, 5.23\%.

4.8. General Procedure for Synthesis of New Schiff's Base $(\mathbf{9} \boldsymbol{a}-\boldsymbol{g})$. A mixture of 2-hydroxy-4-pentadecylbenzaldehyde $2(0.01 \mathrm{~mol})$ and various aromatic amines $(0.01 \mathrm{~mol})$ in the presence of glacial acetic acid $(1 \mathrm{~mL})$ in ethanol $(30 \mathrm{~mL})$ was refluxed for $5 \mathrm{~h}$. The completion of the reaction was monitored by TLC. The reaction mixture was allowed to cool down to room temperature and then poured into crushed ice. The precipitate was filtered, dried, and recrystallized from absolute ethanol. The resulting solid was further purified by column chromatography [silica, n-hexane/ethyl acetate $(90: 10)]$ to get pure compounds $\mathbf{9 a}-\mathbf{g}$ (Scheme 4).
4.8.1. 5-Pentadecyl-2-((phenylimino)methyl)phenol (9a). Yield: $65 \%$; mp: $201-203^{\circ} \mathrm{C}$; IR (KBr): $\nu_{\max }\left(\mathrm{cm}^{-1}\right): 3413.01$ (O-H str.), 1619.21 (C=N str.); ${ }^{1} \mathrm{H}-\mathrm{NMR} \delta$ ppm: 12.61 (s, $1 \mathrm{H}$, $\mathrm{OH}), 8.61(\mathrm{~s}, 1 \mathrm{H}, \mathrm{CH}=\mathrm{N}), 7.81(\mathrm{~d}, J=7.8 \mathrm{~Hz}, 2 \mathrm{H}, \mathrm{Ar}-\mathrm{H})$, $7.50-7.55(\mathrm{~m}, 3 \mathrm{H}, \mathrm{Ar}-\mathrm{H}), 7.25(\mathrm{~d}, J=7.7 \mathrm{~Hz}, 1 \mathrm{H}, \mathrm{Ar}-\mathrm{H})$, 7.03 (s, 1H, Ar-H), 6.89 (d, J = 7.6 Hz, 1H, Ar-H), 2.55 (t, $\left.J=7.6 \mathrm{~Hz}, 2 \mathrm{H}, \mathrm{Ar}-\mathrm{CH}_{2}\right), 1.51-1.10\left(\mathrm{~m}, 26 \mathrm{H},\left(\mathrm{CH}_{2}\right)_{13}\right), 0.85$ $\left(\mathrm{t}, J=6.8 \mathrm{~Hz}, 3 \mathrm{H}, \mathrm{CH}_{3}\right) ;{ }^{13} \mathrm{C}-\mathrm{NMR} \delta$ ppm: $163.2,156.2,153.1$, $143.3,129.6,123.2,120.1,119.9,118.8,116.3,115.7$ (aromatic carbons), 35.3, 35.1, 34.8, 31.2, 30.8, 30.5, 30.1, 28.9, 28.8, 28.7, 28.6, 28.5, 22.3, 14.2 (methylene carbons), $13.8\left(\mathrm{CH}_{3}\right)$; $\mathrm{MS}$, $m / z: 406.11\left(\mathrm{M}^{+}\right)$. Anal. calcd. for $\mathrm{C}_{28} \mathrm{H}_{41} \mathrm{NO}$ : C, 82.52; $\mathrm{H}$, 10.12 ; N, 3.44; found: C, $82.24 ; \mathrm{H}, 10.03 ; \mathrm{N}, 3.03 \%$.

4.8.2. 2-((2-Methoxy-4-nitrophenylimino)methyl)-5-pentadecylphenol (9b). Yield: 70\%; mp: $210-212^{\circ} \mathrm{C}$; IR (KBr): $\nu_{\max }$ $\left(\mathrm{cm}^{-1}\right)$ : 3425.01 (O-H str.), 1628.03 (C=N str.); ${ }^{1} \mathrm{H}-\mathrm{NMR}$ $\delta$ ppm: 12.77 (s, 1H, OH), $8.69(\mathrm{~s}, 1 \mathrm{H}, \mathrm{CH}=\mathrm{N}), 8.03(\mathrm{~d}, J=$ $7.8 \mathrm{~Hz}, 1 \mathrm{H}, \mathrm{Ar}-\mathrm{H}), 7.68$ (d, J = 7.7 Hz, 1H, Ar-H), 7.51 (s, 1H, $\mathrm{Ar}-\mathrm{H}), 6.83(\mathrm{~d}, J=7.6 \mathrm{~Hz}, 1 \mathrm{H}, \mathrm{Ar}-\mathrm{H}), 6.79(\mathrm{~d}, J=7.7 \mathrm{~Hz}, 1 \mathrm{H}$, $\mathrm{Ar}-\mathrm{H}), 3.81\left(\mathrm{~s}, 3 \mathrm{H}, \mathrm{OCH}_{3}\right), 2.63\left(\mathrm{t}, \mathrm{J}=7.6 \mathrm{~Hz}, 2 \mathrm{H}, \mathrm{Ar}-\mathrm{CH}_{2}\right.$ ), $1.55-1.13\left(\mathrm{~m}, 26 \mathrm{H},\left(\mathrm{CH}_{2}\right)_{13}\right), 0.83\left(\mathrm{t}, J=6.8 \mathrm{~Hz}, 3 \mathrm{H}, \mathrm{CH}_{3}\right)$; ${ }^{13} \mathrm{C}-\mathrm{NMR} \delta$ ppm: 160.7, 157.2, 152.1, 143.6, 129.8, 128.9, 120.1, $119.9,118.4$, 116.1, 115.9, 114.6, 112.5 (aromatic carbons), 50.6, 35.3, 35.1, 34.8, 31.2, 30.8, 30.1, 28.9, 28.8, 28.7, 28.6, 28.5, 28.2, 22.0, 13.8 (methylene carbons), $13.5\left(\mathrm{CH}_{3}\right) ; \mathrm{MS}, \mathrm{m} / z: 482.97$ $\left(\mathrm{M}^{+}\right)$. Anal. calcd. for $\mathrm{C}_{29} \mathrm{H}_{42} \mathrm{~N}_{2} \mathrm{O}_{4}$ : C, 72.17; $\mathrm{H}, 8.77 ; \mathrm{N}, 5.80$; found: C, 71.94; H, 8.55; N, 5.65\%.

4.8.3. 2-((2,4-Dinitrophenylimino)methyl)-5-pentadecylphenol (9c). Yield: 68\%; mp: $191-193^{\circ} \mathrm{C}$; IR $(\mathrm{KBr}): v_{\max }\left(\mathrm{cm}^{-1}\right)$ : 3398.15 (O-H str.), 1619.03 (C=N str.); ${ }^{1} \mathrm{H}-\mathrm{NMR} \delta$ ppm: 12.24 (s, 1H, OH), 9.02 (s, 1H, Ar-H), 8.70 (d, J = 7.7 Hz, 1H, Ar$\mathrm{H}), 8.60(\mathrm{~s}, 1 \mathrm{H}, \mathrm{CH}=\mathrm{N}), 8.38(\mathrm{~d}, J=7.7 \mathrm{~Hz}, 1 \mathrm{H}, \mathrm{Ar}-\mathrm{H})$, $7.12(\mathrm{~d}, J=7.8 \mathrm{~Hz}, 1 \mathrm{H}, \mathrm{Ar}-\mathrm{H}), 7.09$ (s, 1H, Ar-H), 6.91 (d, $J=7.6 \mathrm{~Hz}, 1 \mathrm{H}, \mathrm{Ar}-\mathrm{H}), 2.64\left(\mathrm{t}, J=7.6 \mathrm{~Hz}, 2 \mathrm{H}, \mathrm{Ar}-\mathrm{CH}_{2}\right)$, $1.58-1.12\left(\mathrm{~m}, 26 \mathrm{H},\left(\mathrm{CH}_{2}\right)_{13}\right), 0.83\left(\mathrm{t}, J=6.8 \mathrm{~Hz}, 3 \mathrm{H}, \mathrm{CH}_{3}\right)$; ${ }^{13}$ C-NMR $\delta$ ppm: 161.4, 158.2, 156.1, 145.6, 129.8, 128.9, 123.1, $119.9,118.8,116.5,114.9,114.7,112.5$ (aromatic carbons), 35.3, 35.1, 34.8, 31.2, 30.8, 30.1, 28.9, 28.8, 28.7, 28.6, 28.5, 22.0, 14.2 (methylene carbons), $13.7\left(\mathrm{CH}_{3}\right) ; \mathrm{MS}, \mathrm{m} / z: 496.11\left(\mathrm{M}^{+}+1\right)$. Anal. calcd. for $\mathrm{C}_{28} \mathrm{H}_{39} \mathrm{~N}_{3} \mathrm{O}_{5}: \mathrm{C}, 67.58 ; \mathrm{H}, 7.90 ; \mathrm{N}, 8.44$; found: C, 67.12; H, 7.41; N, 7.13\%.

4.8.4. 2-((2-Chloro-4-nitrophenylimino)methyl)-5-pentadecylphenol (9d). Yield: 90\%; mp: $165-167^{\circ} \mathrm{C}$; IR (KBr): $v_{\max }$ $\left(\mathrm{cm}^{-1}\right)$ : 3415.29 (O-H str.), 1626.09 (C=N str.); ${ }^{1} \mathrm{H}-\mathrm{NMR}$ $\delta$ ppm: $12.71(\mathrm{~s}, 1 \mathrm{H}, \mathrm{OH}), 8.80(\mathrm{~s}, 1 \mathrm{H}, \mathrm{CH}=\mathrm{N}), 8.25(\mathrm{~s}, 1 \mathrm{H}, \mathrm{Ar}-$ $\mathrm{H}), 8.05(\mathrm{~d}, J=7.8 \mathrm{~Hz}, 1 \mathrm{H}, \mathrm{Ar}-\mathrm{H}), 7.85(\mathrm{~d}, J=7.6 \mathrm{~Hz}, 1 \mathrm{H}$, Ar-H), 7.82 (d, J = 7.8 Hz, 1H, Ar-H), 7.05 (s, 1H, Ar-H), 6.93 $(\mathrm{d}, J=7.6 \mathrm{~Hz}, 1 \mathrm{H}, \mathrm{Ar}-\mathrm{H}), 2.67\left(\mathrm{t}, J=7.6 \mathrm{~Hz}, 2 \mathrm{H}, \mathrm{Ar}-\mathrm{CH}_{2}\right)$, $1.55-1.13\left(\mathrm{~m}, 26 \mathrm{H},\left(\mathrm{CH}_{2}\right)_{13}\right), 0.82\left(\mathrm{t}, J=6.8 \mathrm{~Hz}, 3 \mathrm{H}, \mathrm{CH}_{3}\right)$; ${ }^{13} \mathrm{C}-\mathrm{NMR} \delta$ ppm: 163.1, 159.7, 152.1, 143.6, 129.8, 128.9, 120.1, $119.9,118.4,116.5,115.4,114.7,112.6$ (aromatic carbons), 35.3, 35.1, 34.8, 31.2, 30.8, 30.1, 28.9, 28.8, 28.7, 28.6, 28.5, 22.0, 13.8 (methylene carbons), $12.5\left(\mathrm{CH}_{3}\right)$; $\mathrm{MS}, \mathrm{m} / z$ : $485.23\left(\mathrm{M}^{+}\right)$. Anal. calcd. for $\mathrm{C}_{28} \mathrm{H}_{39} \mathrm{ClN}_{2} \mathrm{O}_{3}: \mathrm{C}, 69.04 ; \mathrm{H}, 8.07 ; \mathrm{N}, 5.75$; found: $\mathrm{C}$, $68.94 ; \mathrm{H}, 7.98 ; \mathrm{N}, 5.68 \%$. 


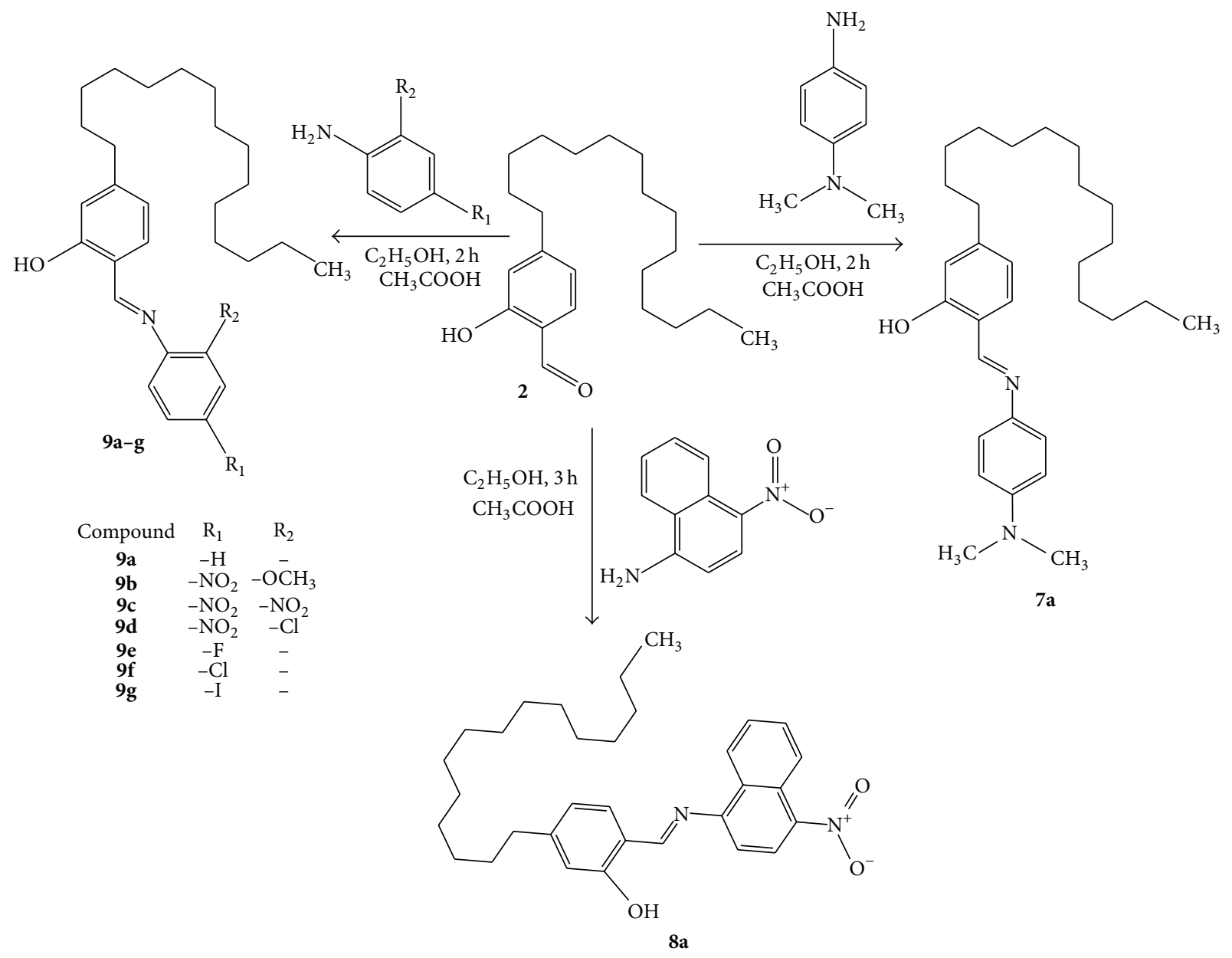

Scheme 4: Synthesis of novel Schiff base series of compounds.

4.8.5. 2-((4-Fluorophenylimino)methyl)-5-pentadecylphenol (9e). Yield: $76 \%$; mp: $209-211^{\circ} \mathrm{C}$; IR $(\mathrm{KBr}): \nu_{\max }\left(\mathrm{cm}^{-1}\right)$ : 3434.15 (O-H str.), 1621.31 (C=N str.); ${ }^{1} \mathrm{H}-\mathrm{NMR} \delta$ ppm: 12.10 $(\mathrm{s}, 1 \mathrm{H}, \mathrm{OH}), 8.79(\mathrm{~s}, 1 \mathrm{H}, \mathrm{CH}=\mathrm{N}), 7.60(\mathrm{~d}, J=7.8 \mathrm{~Hz}, 1 \mathrm{H}$, Ar-H), 7.51 (d, $J=7.7 \mathrm{~Hz}, 2 \mathrm{H}, \operatorname{Ar}-\mathrm{H}), 7.47(\mathrm{~d}, J=7.7 \mathrm{~Hz}$, $2 \mathrm{H}, \operatorname{Ar}-\mathrm{H}), 7.01$ (s, $1 \mathrm{H}, \operatorname{Ar}-\mathrm{H}), 6.89(\mathrm{~d}, J=7.6 \mathrm{~Hz}, 1 \mathrm{H}$, $\mathrm{Ar}-\mathrm{H}), 2.64$ (t, $\left.\mathrm{J}=7.6 \mathrm{~Hz}, 2 \mathrm{H}, \mathrm{Ar}-\mathrm{CH}_{2}\right), 1.56-1.13(\mathrm{~m}, 26 \mathrm{H}$, $\left.\left(\mathrm{CH}_{2}\right)_{13}\right), 0.81\left(\mathrm{t}, J=6.8 \mathrm{~Hz}, 3 \mathrm{H}, \mathrm{CH}_{3}\right) ;{ }^{13} \mathrm{C}-\mathrm{NMR} \delta \mathrm{ppm}$ : $163.4,158.2,153.1,143.6,129.3,128.9,120.1,119.9,118.8,116.5$, 115.4 (aromatic carbons), 35.3, 35.1, 34.8, 31.2, 30.8, 30.1, 28.9, 28.8, 28.7, 28.6, 28.5, 22.0, 13.8 (methylene carbons), 13.5 $\left(\mathrm{CH}_{3}\right)$; MS, $m / z$ : $424.04\left(\mathrm{M}^{+}\right)$. Anal. calcd. for $\mathrm{C}_{28} \mathrm{H}_{40} \mathrm{FNO}$ : C, 79.01; H, 9.47; N, 3.29; found: C, 78.94; H, 9.44; N, 3.13\%.

4.8.6. 2-((4-Chlorophenylimino)methyl)-5-pentadecylphenol (9f). Yield: $65 \%$; mp: $205-207^{\circ} \mathrm{C}$; IR $(\mathrm{KBr}): \nu_{\max }\left(\mathrm{cm}^{-1}\right)$ : 3408.31 (O-H str.), 1618.09 (C=N str.); ${ }^{1} \mathrm{H}-\mathrm{NMR} \delta$ ppm: 12.89 (s, $1 \mathrm{H}, \mathrm{OH}), 8.81$ (s, $1 \mathrm{H}, \mathrm{CH}=\mathrm{N}), 7.72$ (d, $J=7.8 \mathrm{~Hz}, 1 \mathrm{H}$, $\mathrm{Ar}-\mathrm{H}), 7.42(\mathrm{~d}, J=7.7 \mathrm{~Hz}, 2 \mathrm{H}, \mathrm{Ar}-\mathrm{H}), 7.09$ (d, $J=7.7 \mathrm{~Hz}$, $2 \mathrm{H}, \mathrm{Ar}-\mathrm{H}), 6.99$ (s, 1H, Ar-H), 6.89 (d, J = $7.8 \mathrm{~Hz}, 1 \mathrm{H}$, $\mathrm{Ar}-\mathrm{H}), 2.63\left(\mathrm{t}, \mathrm{J}=7.6 \mathrm{~Hz}, 2 \mathrm{H}, \mathrm{Ar}-\mathrm{CH}_{2}\right), 1.55-1.13(\mathrm{~m}, 26 \mathrm{H}$, $\left.\left(\mathrm{CH}_{2}\right)_{13}\right), 0.80\left(\mathrm{t}, J=6.8 \mathrm{~Hz}, 3 \mathrm{H}, \mathrm{CH}_{3}\right) ;{ }^{13} \mathrm{C}-\mathrm{NMR} \delta \mathrm{ppm}$ : $163.3,157.1,156.4,147.2,129.8,128.9,120.1,119.9,118.8,116.8$,
116.3 (aromatic carbons), 35.3, 35.1, 34.8, 31.2, 30.8, 30.1, 28.9, 28.8, 28.7, 28.6, 28.5, 26.5, 22.0, 13.8 (methylene carbons), 12.8 $\left(\mathrm{CH}_{3}\right)$; MS, $m / z: 440.21\left(\mathrm{M}^{+}\right)$. Anal. calcd. for $\mathrm{C}_{28} \mathrm{H}_{40} \mathrm{ClNO}$ : C, 76.07; H, 9.12; N, 3.17; found: C, 75.94; H, 8.99; N, 3.03\%.

4.8.7. 2-((4-Iodophenylimino)methyl)-5-pentadecylphenol (9g). Yield: $61 \%$; mp: $179-181^{\circ} \mathrm{C}$; IR $(\mathrm{KBr}): v_{\max }\left(\mathrm{cm}^{-1}\right)$ : 3435.23 (O-H str.), 1624.07 (C=N str.); ${ }^{1} \mathrm{H}-\mathrm{NMR} \delta$ ppm: 12.83 (s, 1H, OH), 8.89 (s, $1 \mathrm{H}, \mathrm{CH}=\mathrm{N}), 7.77$ (d, $J=7.8 \mathrm{~Hz}, 1 \mathrm{H}$, Ar-H), 7.52 (d, $J=7.7 \mathrm{~Hz}, 2 \mathrm{H}, \mathrm{Ar}-\mathrm{H}), 7.20$ (d, $J=7.7 \mathrm{~Hz}$, $2 \mathrm{H}, \mathrm{Ar}-\mathrm{H}), 6.80(\mathrm{~d}, J=7.6 \mathrm{~Hz}, 1 \mathrm{H}, \mathrm{Ar}-\mathrm{H}), 6.77(\mathrm{~s}, 1 \mathrm{H}$, $\mathrm{Ar}-\mathrm{H}), 2.63$ (t, $\left.J=7.6 \mathrm{~Hz}, 2 \mathrm{H}, \mathrm{Ar}-\mathrm{CH}_{2}\right), 1.56-1.13(\mathrm{~m}, 26 \mathrm{H}$, $\left.\left(\mathrm{CH}_{2}\right)_{13}\right), 0.83\left(\mathrm{t}, \mathrm{J}=6.8 \mathrm{~Hz}, 3 \mathrm{H}, \mathrm{CH}_{3}\right) ;{ }^{13} \mathrm{C}-\mathrm{NMR} \delta \mathrm{ppm}$ : $161.2,157.4,152.8,143.2,129.7,128.9,120.1,119.9,118.8,117.5$, 117.2 (aromatic carbons), 35.3, 35.1, 34.8, 31.2, 30.8, 30.1, 28.9, 28.8, 28.7, 28.6, 28.5, 25.5, 22.2, 13.6 (methylene carbons), 12.8 $\left(\mathrm{CH}_{3}\right)$; MS, $m / z: 533.72\left(\mathrm{M}^{+}\right)$. Anal. calcd. for $\mathrm{C}_{28} \mathrm{H}_{40} \mathrm{INO}$ : C, 63.03; H, 7.56; N, 2.63; found: C, 62.86; H, 7.72; N, 2.83\%.

4.9. Synthesis of 2-((4'-Aminobiphenyl-4-ylimino)methyl)-5pentadecylphenol (10a). A mixture of compound 2 (3.30 g, $0.01 \mathrm{~mol})$ and biphenyl- $4,4^{\prime}$-diamine $(1.84 \mathrm{~g}, 0.010 \mathrm{~mol})$ in the presence of acetic acid in ethanol was refluxed for $4 \mathrm{~h}$. The 


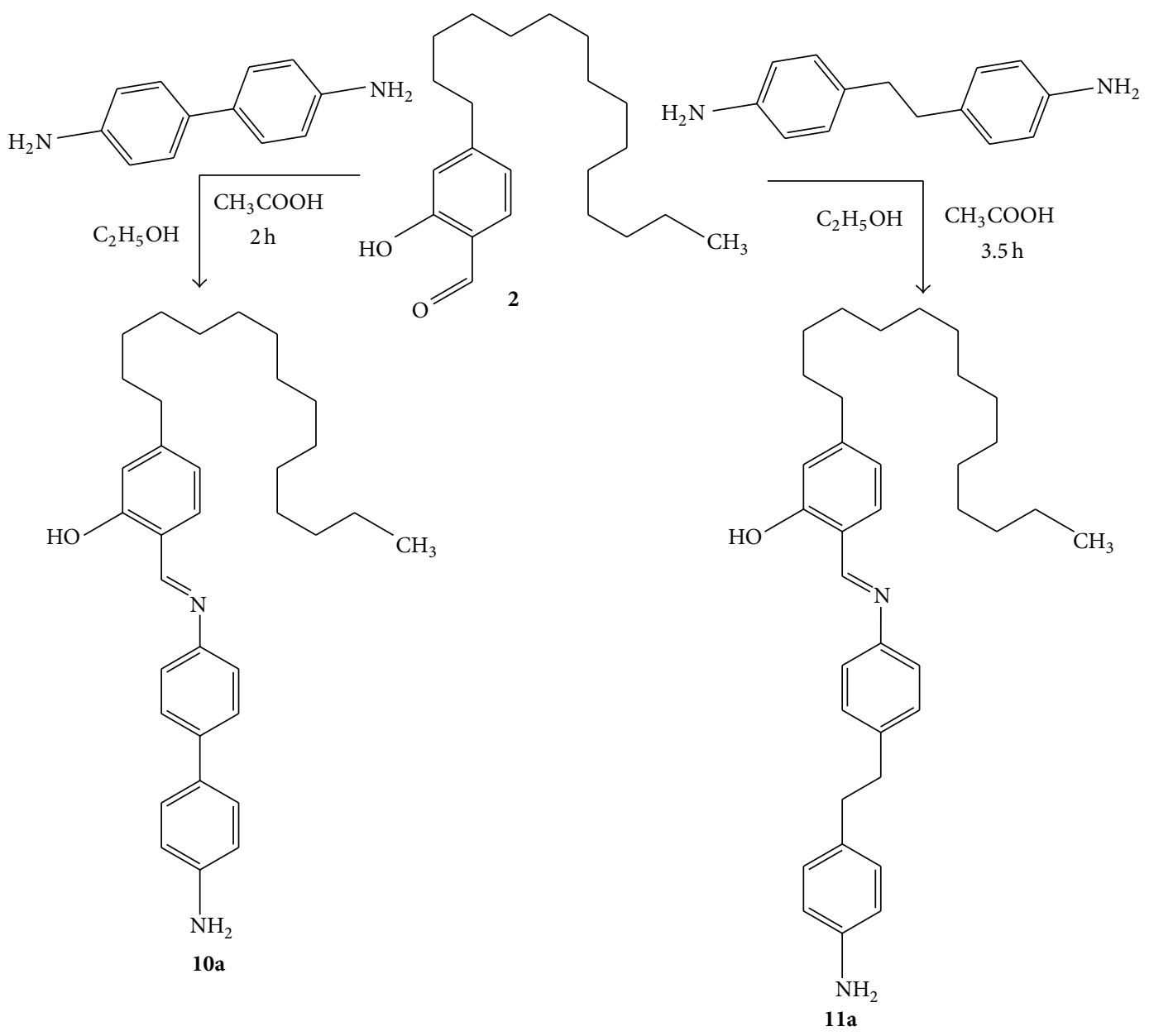

Scheme 5: Special new Schiff base compounds.

reaction mixture was poured into crushed ice. The precipitate was filtered, dried, and recrystallized from ethanol to get pure compound 10a, no need for further purification (Scheme 5).

Yield: $73 \%$; mp: $301-303^{\circ} \mathrm{C}$; IR $(\mathrm{KBr}): \nu_{\max }\left(\mathrm{cm}^{-1}\right)$ : 3434.03 (O-H str.), 1616.32 (C=N str.); ${ }^{1} \mathrm{H}-\mathrm{NMR} \delta$ ppm: 13.12 (s, $1 \mathrm{H}, \mathrm{OH}), 8.93(\mathrm{~s}, 1 \mathrm{H}, \mathrm{CH}=\mathrm{N}), 7.63(\mathrm{~d}, J=7.7 \mathrm{~Hz}, 2 \mathrm{H}$, $\mathrm{Ar}-\mathrm{H}), 7.61$ (d, J = 7.8 Hz, 1H, Ar-H), 7.49 (m, 4H, Ar-H), $7.09(\mathrm{~d}, J=7.8 \mathrm{~Hz}, 1 \mathrm{H}, \mathrm{Ar}-\mathrm{H}), 6.89$ (s, 1H, Ar-H), $6.56(\mathrm{~d}$, $J=7.7 \mathrm{~Hz}, 2 \mathrm{H}, \mathrm{Ar}-\mathrm{H}), 5.15\left(\mathrm{~s}, \mathrm{NH}_{2}\right), 2.69(\mathrm{t}, J=7.6 \mathrm{~Hz}, 2 \mathrm{H}$, $\left.\mathrm{Ar}-\mathrm{CH}_{2}\right), 1.57-1.13\left(\mathrm{~m}, 26 \mathrm{H},\left(\mathrm{CH}_{2}\right)_{13}\right), 0.89(\mathrm{t}, J=6.8 \mathrm{~Hz}, 3 \mathrm{H}$, $\left.\mathrm{CH}_{3}\right) ;{ }^{13} \mathrm{C}-\mathrm{NMR} \delta$ ppm: $162.3,157.9,153.1,143.8,129.9,128.4$, 121.1, 119.1, 118.8, 117.5, 117.3, 116.4, 115.3, 114.7, 112.5 (aromatic carbons), 35.3, 35.1, 34.8, 31.2, 30.8, 30.1, 29.5, 28.9, 28.8, 28.7, 28.6, 28.5, 22.0, 13.8 (methylene carbons), $13.3\left(\mathrm{CH}_{3}\right)$; MS, $m / z$ : $497.45\left(\mathrm{M}^{+}\right)$. Anal. calcd. for $\mathrm{C}_{34} \mathrm{H}_{46} \mathrm{~N}_{2} \mathrm{O}$ : C, 81.88; $\mathrm{H}$, 9.30; N, 5.62; found: C, 81.49; H, 9.07; N, 5.33\%.

4.10. Synthesis of 2-((4-(4-Aminophenethyl)phenylimino)methyl)-5-pentadecylphenol (11a). A mixture of compound 2 $(3.30 \mathrm{~g}, 0.01 \mathrm{~mol})$ and 4,4-ethane-1,2-diyldianiline $(2.12 \mathrm{~g}$, $0.01 \mathrm{~mol}$ ) in the presence of glacial acetic acid in absolute ethanol $(20 \mathrm{~mL})$ was heated under reflux for $3 \mathrm{~h}$. After completion of the reaction, the reaction mixture was poured into crushed ice. The precipitate was filtered, dried, and recrystallized from ethanol to get compound 11a.

Yield: $81 \%$; mp: $189-191^{\circ} \mathrm{C}$; IR (KBr): $\nu_{\max }\left(\mathrm{cm}^{-1}\right): 3435.31$ (O-H str.), 1624.63 (C=N str.); ${ }^{1} \mathrm{H}-\mathrm{NMR} \delta$ ppm: 12.80 (s, $1 \mathrm{H}$, $\mathrm{OH}), 8.89$ (s, 1H, CH=N), $7.63(\mathrm{~d}, J=7.8 \mathrm{~Hz}, 1 \mathrm{H}, \mathrm{Ar}-\mathrm{H}), 7.41$ $(\mathrm{d}, J=7.6 \mathrm{~Hz}, 2 \mathrm{H}, \mathrm{Ar}-\mathrm{H}), 7.32(\mathrm{~d}, J=7.6 \mathrm{~Hz}, 2 \mathrm{H}, \mathrm{Ar}-\mathrm{H})$, $7.05(\mathrm{~d}, J=7.6 \mathrm{~Hz}, 2 \mathrm{H}, \mathrm{Ar}-\mathrm{H}), 7.01(\mathrm{~d}, J=7.8 \mathrm{~Hz}, 1 \mathrm{H}, \mathrm{Ar}-$ H), 6.88 (s, 1H, Ar-H), 6.68 (d, J = 7.6 Hz, 2H, Ar-H), 5.25 $\left(\mathrm{s}, \mathrm{NH}_{2}\right), 2.84\left(\mathrm{~s},-\mathrm{CH}_{2}-\mathrm{CH}_{2}-\right), 2.65(\mathrm{t}, J=7.6 \mathrm{~Hz}, 2 \mathrm{H}, \mathrm{Ar}-$ $\left.\mathrm{CH}_{2}\right), 1.57-1.13\left(\mathrm{~m}, 26 \mathrm{H},\left(\mathrm{CH}_{2}\right)_{13}\right), 0.83(\mathrm{t}, J=6.8 \mathrm{~Hz}, 3 \mathrm{H}$, $\left.\mathrm{CH}_{3}\right) ;{ }^{13} \mathrm{C}-\mathrm{NMR} \delta$ ppm: 163.0, 157.8, 152.2, 143.6, 129.8, 128.9, $120.1,119.9,118.8,117.5,117.8,116.5,115.0,114.7,112.5$ (aromatic carbons), 37.4, 35.3, 35.1, 34.8, 31.2, 30.8, 30.1, 28.9, 28.8, 28.7, 28.6, 28.5, 22.0, 20.3, 15.8 (methylene carbons), $14.2\left(\mathrm{CH}_{3}\right)$; MS, $m / z: 526.39\left(\mathrm{M}^{+}\right)$. Anal. calcd. for $\mathrm{C}_{36} \mathrm{H}_{50} \mathrm{~N}_{2} \mathrm{O}: \mathrm{C}, 82.08$; $\mathrm{H}, 9.57$; N, 5.32; found: C, 82.12; H, 9.40; N, 5.13\%.

\section{Conclusion}

In conclusion, a new series of 6-substitued-3-chloro- $\mathrm{N}^{\prime}-(2-$ hydroxy-4-pentadecylbenzylidene)benzothiophene-2-carbohydrazide derivatives were synthesized, fully characterized, and evaluated for their antibacterial and antifungal 
activities. The newly synthesized compounds exhibited low to moderate antibacterial activity against $S$. aureus and $B$. subtilis andsignificant antifungal activity against $C$. albicans and $C$. pannical. It can beconcluded that these classes of compounds certainly hold great promise towards good active leads in medicinal chemistry. A further study to acquire more information concerning pharmacological activity is in progress.

\section{Conflict of Interests}

The authors declare that there is no conflict of interests regarding the publication of this paper.

\section{Acknowledgments}

This work was supported by the Higher Education Research Promotion and National Research University Project of Thailand, Office of the Higher Education Commission. The postdoctoral fellowship grant from the URC, University of Johannesburg, South Africa, was gratefully acknowledged.

\section{References}

[1] P. Panneerselvam, B. A. Rather, D. R. S. Reddy, and N. R. Kumar, "Synthesis and anti-microbial screening of some Schiff bases of 3-amino-6,8-dibromo-2-phenylquinazolin-4(3H)-ones," European Journal of Medicinal Chemistry, vol. 44, no. 5, pp. 23282333, 2009.

[2] X. Jin, J. Wang, and J. Bai, "Synthesis and antimicrobial activity of the Schiff base from chitosan and citral," Carbohydrate Research, vol. 344, no. 6, pp. 825-829, 2009.

[3] S. K. Bharti, G. Nath, R. Tilak, and S. K. Singh, "Synthesis, antibacterial and anti-fungal activities of some novel Schiff bases containing 2,4-disubstituted thiazole ring," European Journal of Medicinal Chemistry, vol. 45, no. 2, pp. 651-660, 2010.

[4] G. B. Bagihalli, P. G. Avaji, S. A. Patil, and P. S. Badami, "Synthesis, spectral characterization, in vitro antibacterial, antifungal and cytotoxic activities of $\mathrm{Co}(\mathrm{II}), \mathrm{Ni}(\mathrm{II})$ and $\mathrm{Cu}(\mathrm{II})$ complexes with 1,2,4-triazole Schiff bases," European Journal of Medicinal Chemistry, vol. 43, no. 12, pp. 2639-2649, 2008.

[5] Y. C. Liu and Z. Y. Yang, "Crystal structures, antioxidation and DNA binding properties of Eu(III) complexes with Schiff-base ligands derived from 8-hydroxyquinoline-2-carboxyaldehyde and three aroylhydrazines," Journal of Inorganic Biochemistry, vol. 103, no. 7, pp. 1014-1022, 2009.

[6] H. Nawaz, Z. Akhter, S. Yameen, H. M. Siddiqi, B. Mirza, and A. Rifat, "Synthesis and biological evaluations of some Schiffbase esters of ferrocenyl aniline and simple aniline," Journal of Organometallic Chemistry, vol. 694, no. 14, pp. 2198-2203, 2009.

[7] S. T. Ha, L. K. Ong, J. P. W. Wong et al., "Mesogenic Schiff's base ether with dimethylamino end group," Phase Transitions, vol. 82, no. 5, pp. 387-397, 2009.

[8] S. T. Ha, L. K. Ong, S. T. Ong et al., "Synthesis and mesomorphic properties of new Schiff base esters with different alkyl chains," Chinese Chemical Letters, vol. 20, no. 7, pp. 767-770, 2009.

[9] S.-T. Ha, T.-M. Koh, S.-T. Ong, J.-K. Beh, and L.-K. Ong, "Synthesis of a new liquid crystal, 3-hydroxy-4-[(6-methoxy-1,3benzothiazol-2-yl)imino]methylphenyl palmitate," Molbank, vol. 2009, no. 3, article M608, 2009.
[10] S. T. Ha, L. K. Ong, Y. F. Win, and T. M. Koh, “4-[(Pyridin3-ylmethylene)amino]phenyltetradecanoate," Molbank, no. 1, Article ID M585, 2009.

[11] S. T. Ha, L. K. Ong, Y. F. Win, T. M. Koh, and G.-Y. Yeap, "4-[(Pyridin-3-ylmethylene)amino]phenylhexadecanoate," Molbank, no. 1, article M584, 2009.

[12] P. Payne, J. H. P. Tyman, S. K. Mehet, and A. Ninagawa, "The synthesis of 2-hydroxymethyl derivatives of phenols," Journal of Chemical Research, vol. 2006, no. 6, pp. 402-405, 2006.

[13] S. Parkey and N. Castle, "The synthesis of dimethoxy[1]benzothieno[2,3-c] quinolines," Journal of Heterocyclic Chemistry, vol. 23, no. 5, pp. 1571-1579, 1986.

[14] British Pharmacopoeia, A300, Vol. IV, Appendix XIV, 2005.

[15] J. G. Vincent and H. W. Vincent, "Filter paper disc modification of oxford cup peneciline cup determination," Proceedings of the Society for Experimental Biology and Medicine, vol. 55, pp. 162$164,1944$. 

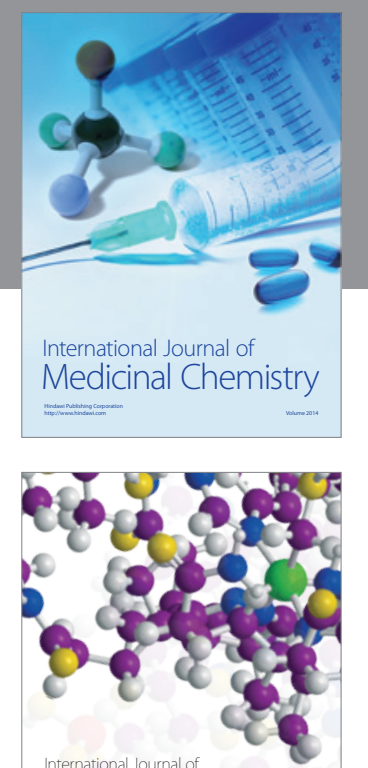

\section{Carbohydrate} Chemistry

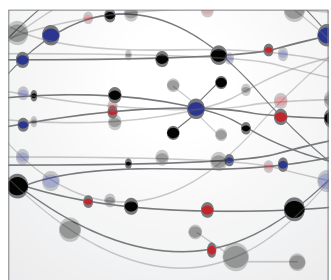

The Scientific World Journal
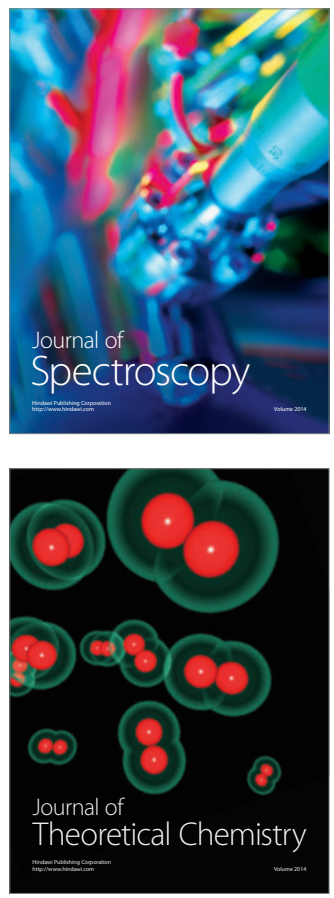
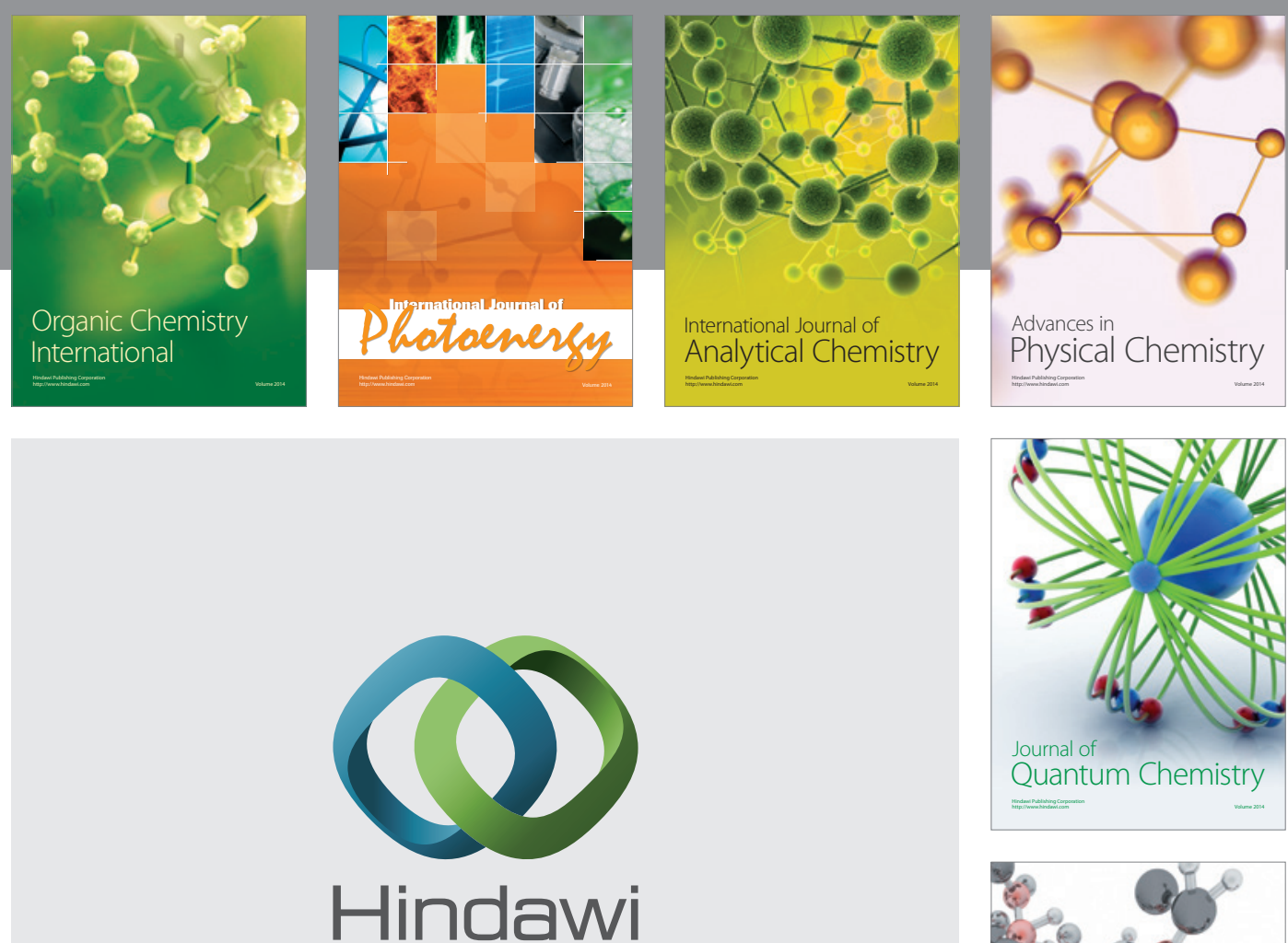

Submit your manuscripts at

http://www.hindawi.com

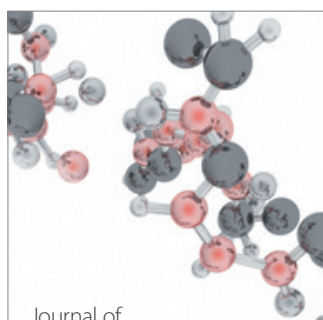

Analytical Methods

in Chemistry

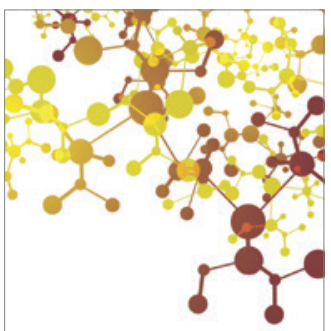

Journal of

Applied Chemistry

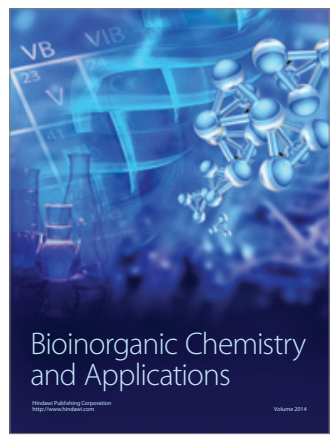

Inorganic Chemistry
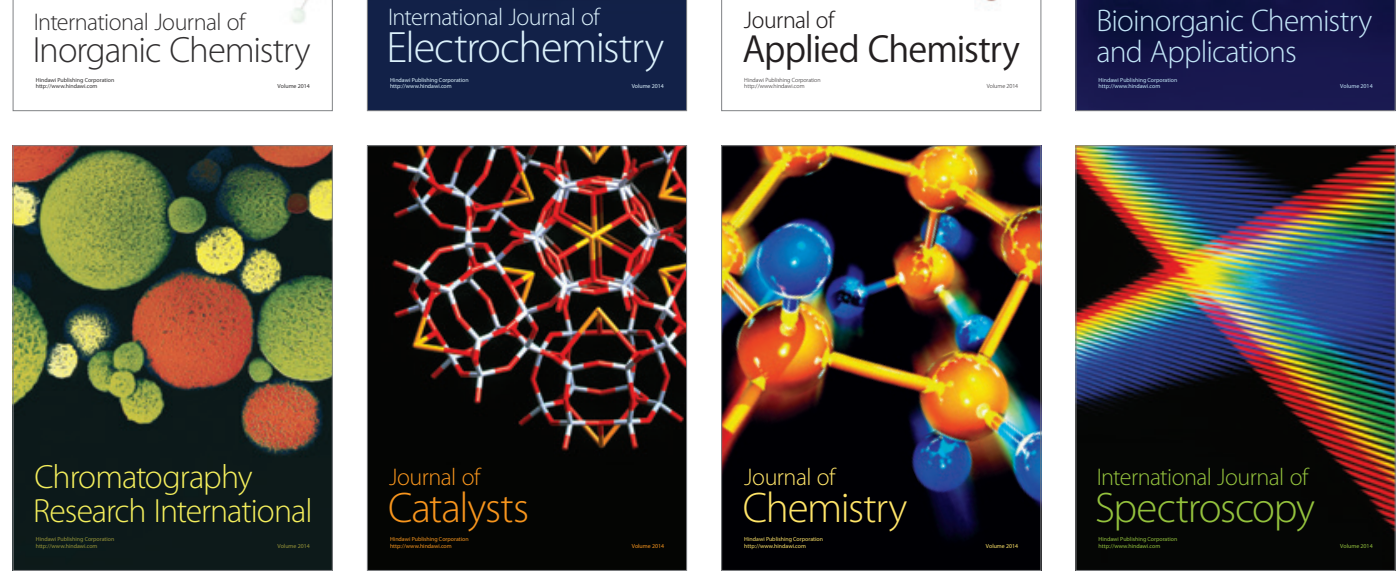